\title{
Review Article \\ Three-Dimensional Graphene-Based Nanomaterials as Electrocatalysts for Oxygen Reduction Reaction
}

\author{
Xuan Ji, ${ }^{1}$ Xin Zhang, ${ }^{2}$ and Xianwen Zhang $^{3}$ \\ ${ }^{1}$ Shanxi Research Institute of Applied Chemistry, Taiyuan 030027, China \\ ${ }^{2}$ Department of Chemical Engineering, Texas Tech University, 6th Street and Canton Avenue, Lubbock, TX 79409, USA \\ ${ }^{3}$ Institute of Advanced Energy Technology and Equipment, Hefei University of Technology, 193rd Tunxi Road, Hefei 230009, China
}

Correspondence should be addressed to Xin Zhang; xin.zhang@ttu.edu and Xianwen Zhang; xianwen.zhang@ttu.edu

Received 14 May 2015; Accepted 15 July 2015

Academic Editor: Nay Ming Huang

Copyright (C) 2015 Xuan Ji et al. This is an open access article distributed under the Creative Commons Attribution License, which permits unrestricted use, distribution, and reproduction in any medium, provided the original work is properly cited.

In recent years, three-dimensional (3D) graphene-based nanomaterials have been demonstrated to be efficient and promising electrocatalysts for oxygen reduction reaction (ORR) in fuel cells application. This review summarizes and categorizes the recent progress on the preparation and performance of these novel materials as ORR catalysts, including heteroatom-doped 3D graphene network, metal-free 3D graphene-based nanocomposites, nonprecious metal-containing 3D graphene-based nanocomposites, and precious metal-containing 3D graphene-based nanocomposites. The challenges and future perspective of this field are also discussed.

\section{Introduction}

As a novel nanomaterial, graphene is a one-atom-thick carbon sheet with a hexagonal packed lattice structure. Since the first direct observation and characterization of a mechanically exfoliated graphene by Geim and Novoselov in 2004, this "miracle material" has attracted tremendous attention and research interest owing to its many unique properties, such as extraordinarily high in-plane electrical mobility, thermal conductivity, mechanical strength, and ultralarge specific surface area [1-4]. Over the past decade, graphene and graphene-based nanomaterials as gifted carbonaceous materials have found a multifunctional application in the fields of photocatalysis, electrocatalysis, water treatment, electrochemical sensor, optical sensor, fiber optic sensor, and so on [5-18]. Among these applications, graphene-based nanomaterials have been actively studied as promising electrode materials in electrochemical devices such as supercapacitors, rechargeable lithium-ion batteries, fuel cells, and solar cells [19-23]. In these studies, graphene may play the role of either the conducting substrate or electroactive center.

Although 2D graphene-based nanomaterials have been demonstrated to be promising in the above electrochemical applications, a huge challenge and need still remain for the efficient use of graphene's large specific surface area and extraordinary electrical, chemical, and mechanical properties. For instance, for electrodes, large surface area and fast electrolyte transfer near electrode surface are required, in order to obtain high rate of electrochemical reaction. However, the graphene sheets on the electrode tend to form irreversible agglomerates or restack due to the strong $\pi-\pi$ stacking and van der Waals interactions between the graphene sheets, which will dramatically reduce the surface area of electrode, hinder the rapid electrolyte diffusion, and consequently limit its practical application in energy devices.

Recently, integrating nanomonolayer graphene into macroscopic 3D porous interconnected networks has attracted significant attention, since the nonagglomerated 3D structure can provide graphene-based nanomaterials with high specific surface areas, strong mechanical strengths, and fast mass and electron transport kinetics due to the combination of $3 \mathrm{D}$ porous structures and the excellent intrinsic properties of graphene [24-29].

On the other hand, the greenhouse gas emissions from the depletion of traditional fossil fuels become the primary cause for global warming and climate change. In this context, increasing demand for sustainable energy has stimulated intense research on energy conversion and storage systems 
that are highly efficient, of low cost, and environmentally friendly. Among the various promising energy conversion technologies, fuel cell, which converts chemical energy from fuels into electricity, is a clean and high-efficiency device with low or zero emissions from operation and has drawn a great deal of attention in terms of both fundamentals and applications [30, 31]. In a fuel cell, the fuel (such as hydrogen, methanol, and ethanol) is oxidized at the anode, and the released electrons are transferred to cathode where oxygen is reduced. Because the oxygen reduction reaction (ORR) at the cathode has sluggish reaction kinetics, which limits the cell performance, the electrocatalysts are necessary to facilitate the ORR. Consequently, the electrocatalyst for the ORR is a key component for fuel cell and plays a vital role in determining the performance of fuel cells, such as power output, open circuit, energy efficiency, and circling life [32$34]$. Over the past few years, various 3D graphene-based nanomaterials have been extensively designed, prepared, and investigated as efficient electrocatalysts for ORR in fuel cells application. However, as far as we know, though it is much needed, a specific and timely review on this area is missing. Accordingly, this review article is provided to categorize and summarize the recent advances in the development of $3 \mathrm{D}$ graphene-based nanomaterials as catalysts for ORR in fuel cells, with focus on introducing and understanding the "preparation-structure-performance" relationship of these novel materials. The challenges and perspective of these emerging materials as ORR catalysts are discussed as well.

\section{Preparation Methods}

In the past several years, tremendous efforts have been devoted to the development of synthetic methods for 3D graphene-based materials with various morphologies, structures, and properties, in order to satisfy the requirements arising from various applications. Considering the existence of several excellent reviews highlighting the classification and summarization of the recent progress in the preparation methods of these materials, the reader should be referred to these reviews to obtain in-depth coverage of the various preparation procedures [35-38]. The subject of the present review is the application of $3 \mathrm{D}$ graphene-based materials in ORR catalysis; thus, only the relevant preparation methods towards this subject are briefly introduced here.

Self-assembly, among the main strategies for the construction of $3 \mathrm{D}$ graphene architecture, is the most promising and widely used strategy to obtain $3 \mathrm{D}$ graphene-based materials as catalysts for ORR. As a typical example, 3D graphene structures can be fabricated through the gelation process of a graphene oxide (GO) suspension followed by reduction to convert GO to reduced graphene oxide (rGO). Both chemical and physical treatments, such as adding various kinds of cross-linkers, changing the $\mathrm{pH}$ value or temperature of the GO suspension, hydrothermal treatment, direct freezedrying, and electrochemical reduction, could trigger the gelation of a GO suspension. Hydrogels, organogels, and aerogels are the main forms of $3 \mathrm{D}$ graphene usually as products of the self-assembly process, and aerogels are obtained from hydrogels and organogels via freeze-drying or supercritical $\mathrm{CO}_{2}$ drying. To create $3 \mathrm{D}$ graphene-based materials, including doped 3D graphene and 3D graphene-based composite, dopant-containing precursor and precursor ions are usually added during or after the GO gelation process followed by reduction for intrinsically doped $3 \mathrm{D}$ graphene and for in situ decoration of NPs/nanomaterials on 3D graphene, respectively. Since GO can be synthesized in large quantities with low cost and easily reduced by different methods, the preparation of $3 \mathrm{D}$ graphene-based materials from GO by self-assembly is a promising and practical way for the mass production of these materials for ORR catalysis.

\section{Applications and Performances of 3D Graphene-Based Nanomaterials as ORR Electrocatalyst}

Although Pt-based electrocatalysts are the most active and popular catalysts for ORR at the cathode in fuel cells, large-scale commercialization is still restricted due to high cost, poor durability, and sluggish electron transfer kinetics. Therefore, the development of alternative catalysts, including metal-free catalysts and nonprecious metal catalysts (NPMC), has drawn tremendous attention in the past decades. In 3D graphene-based electrocatalysts for ORR, 3D graphene may play the role of either inherent and single catalysts such as heteroatom-doped 3D graphene network (discussed in Section 3.1) or supporting materials for other nanocatalysts such as metal-free or metal-containing $3 \mathrm{D}$ graphene-based nanocomposites (discussed in Sections 3.23.4). The following subsections will mainly focus on the preparation and performance of these $3 \mathrm{D}$ graphene-based materials as electrocatalysts for ORR.

3.1. Heteroatom-Doped 3D Graphene Network. More recently, heteroatom-doped carbon nanomaterials (e.g., carbon nanotubes, nanotube cups, ordered mesoporous graphitic arrays, and graphene) have been emerged as a new class of metal-free catalysts or supporting materials for NPMC for ORR [39]. Among these carbon materials, 2D graphene doped with foreign nonmetallic atoms ( $\mathrm{N}$-doped graphene in particular) has been demonstrated both experimentally and theoretically as a promising ORR catalyst owing to its excellent electrocatalytic activities as well as low cost, good durability, and being environmentally friendly [40]. However, the practical applications of 2D graphene as an electrode material in energy device are still limited, because graphene sheets tend to form irreversible agglomerates or restack due to the strong $\pi-\pi$ stacking and van der Waals interactions between the graphene sheets. In this context, the nonagglomerated 3D graphene has been rapidly developed in recent years because its unique structures, which integrate nanomonolayer graphene into macroscopic 3D porous interconnected networks, could effectively translate the excellent intrinsic properties of graphene at the nanoscale into those at the macroscopic level, thus making this doped $3 \mathrm{D}$ graphene as promising catalysts for ORR. 


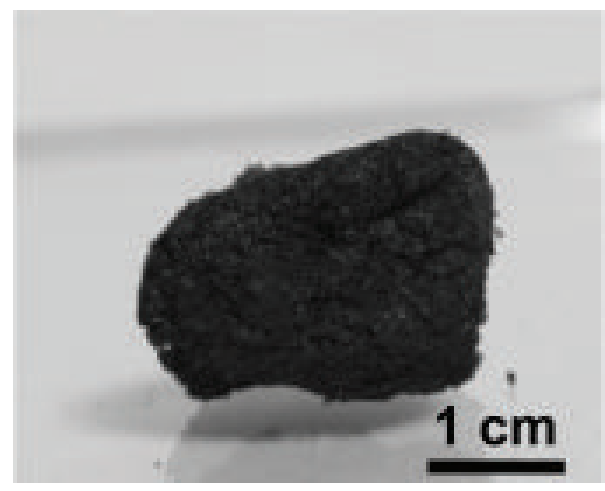

(a)

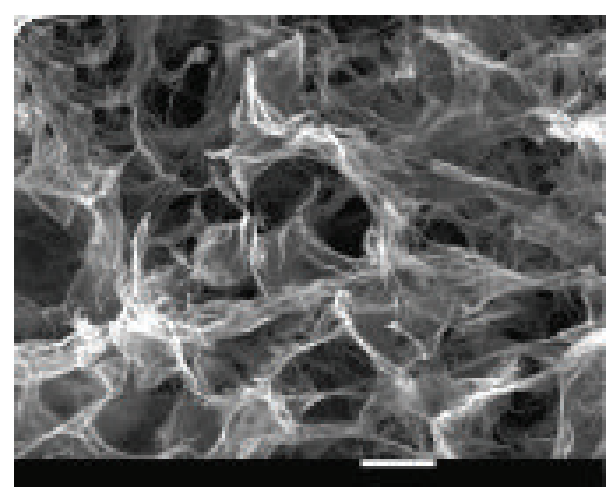

(c)

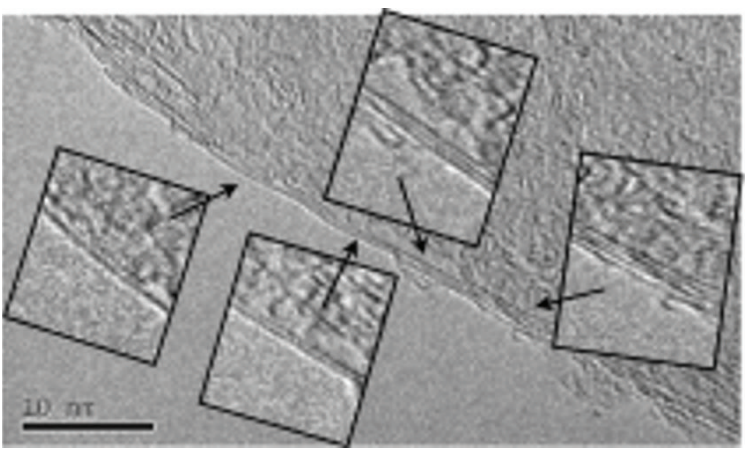

(e)

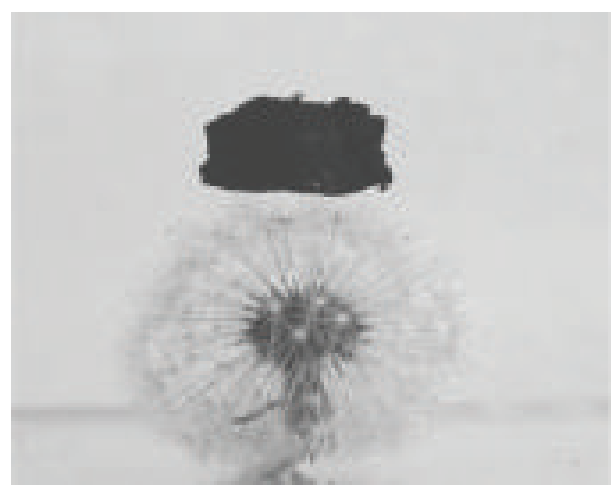

(b)

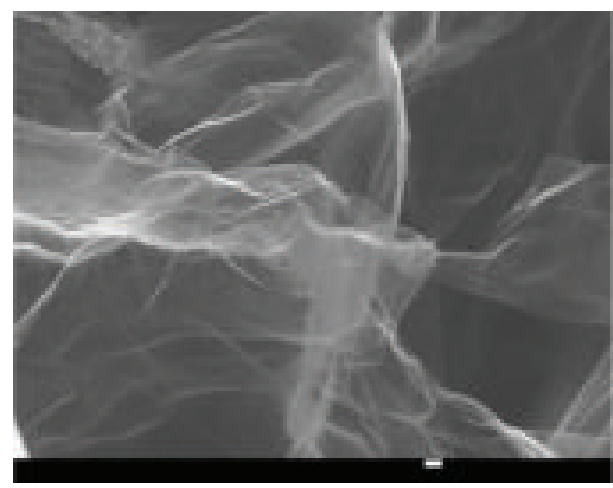

(d)

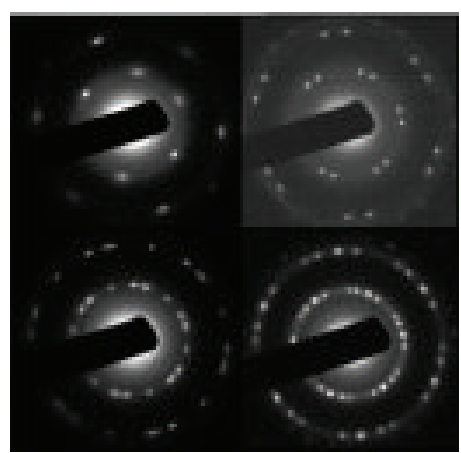

(f)

Figure 1: (a, b) Photographs of an as-prepared superlight GF and one with a piece of GF size of $1.8 \mathrm{~cm} \times 1.1 \mathrm{~cm} \times 1.2 \mathrm{~cm}$ standing on a dandelion. (c, d) SEM images of the sample in (a). (e, f) Typical TEM images of the walls of GF and the corresponding electron diffraction patterns consistent with 1-4 crystalline graphene layers (reprinted from [41]; copyright 2012 WILEY-VCH).

In 2012, Zhao and coworkers developed a method to prepare versatile, $\mathrm{N}$-doped, ultralight $3 \mathrm{D}$ graphene framework (GF), which is demonstrated to be a promising metalfree catalyst for ORR in an alkaline solution (Figures $1(\mathrm{a})$ 1(b)) [41]. The N-doped 3D GF was synthesized through hydrothermal treatment of GO and pyrrole and subsequent freeze-drying and annealing the composition. Different from the conventional 2D stacked graphene film, this doped GF provides a unique 3D pore-rich structure with maximum access to the $\mathrm{N}$ sites within highly exposed graphene sheets and multidimensional electron transport pathways (Figures 1(c)-1(f)). Results from electrochemical measurements showed that this $\mathrm{N}$-containing electrode has high catalytic activity and a four-electron pathway for ORR as compared with that of $\mathrm{Pt} / \mathrm{C}$ electrode. In addition, this $\mathrm{N}$-doped 3D GF exhibited excellent tolerance to the methanol crossover effect and good stability for ORR. Notably, due to its excellent performances in adsorption and capacitance, this $\mathrm{N}$-doped 3D GF also shows a great promise both as a super adsorption material for high-efficiency recyclable sorbent applications and as a new type of electrode material or supporting material for high performance supercapacitors.

In 2013, Su and coworkers developed 3D nitrogen and sulfur codoped graphene frameworks (N/S-GFs) as efficient electrocatalysts under alkaline conditions [42]. These N/S-GFs were prepared by the hydrothermal treatment of 


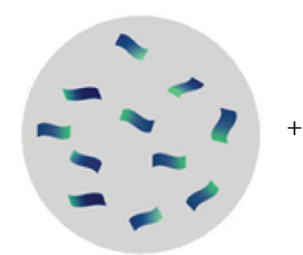

g- $\mathrm{C}_{3} \mathrm{~N}_{4}$ nanosheets in water

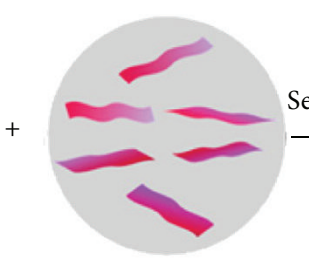

$\mathrm{GO}$ in water

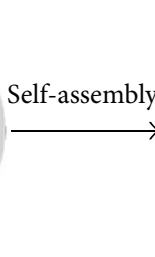

$3 \mathrm{D}$ porous $\mathrm{g}-\mathrm{C}_{3} \mathrm{~N}_{4} / \mathrm{GO}$ architecture

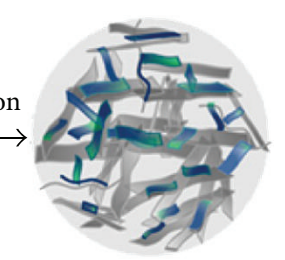

$3 \mathrm{D}$ porous g- $\mathrm{C}_{3} \mathrm{~N}_{4} / \mathrm{rGO}$ architecture

FIGURE 2: Schematic diagram to illustrate the fabrication process of $3 \mathrm{D}$ porous supramolecular architecture of g- $\mathrm{C}_{3} \mathrm{~N}_{4}$ nanosheets and $\mathrm{rGO}$ (reprinted from [44]; copyright 2014 American Chemical Society).

ammonium thiocyanate $\left(\mathrm{NH}_{4} \mathrm{SCN}\right)$ and $\mathrm{GO}$ at $443 \mathrm{~K}$. In the hydrothermal process, $\mathrm{NH}_{4} \mathrm{SCN}$ was decomposed into highly reactive N/S-rich species, such as $\mathrm{NH}_{3}, \mathrm{H}_{2} \mathrm{~S}$, and $\mathrm{CS}_{2}$, which can react with the defective sites and oxygen-containing groups of GO; simultaneously, GO was reduced to graphene and assembled into 3D GFs. The as-prepared N/S-GFs, with large surface area $\left(220 \mathrm{~m}^{2} \mathrm{~g}^{-1}\right.$ BET surface area) and high heteroatom weight content $(12.3 \% \mathrm{~N}$ content and $18.4 \% \mathrm{~S}$ content), were observed to be an interconnected framework of ultrathin graphene nanosheets with porous architecture. According to the study, ORR catalyzed by N/S-GFs was mainly through the four-electron transfer pathway while ORR catalyzed by N-doped GF revealed only two electron transfer mechanism. Moreover, N/S-GFs showed a kineticlimiting current density of $3.9 \mathrm{~mA} / \mathrm{cm}^{2}$, which is comparable to that of commercial $\mathrm{Pt} / \mathrm{C}$ catalyst $\left(4.2 \mathrm{~mA} / \mathrm{cm}^{2}\right)$ and is much higher than $2.8 \mathrm{~mA} / \mathrm{cm}^{2}$ for N-doped GF measured at $-0.36 \mathrm{~V}$. In addition to the high electrocatalytic activity, N/S-GFs also exhibited superior stability and tolerance to methanol crossover effect as compared with those of $\mathrm{Pt} / \mathrm{C}$ catalyst.

Although the aforementioned nonmetallic heteroatomdoped 3D graphene networks are claimed as inherent "metalfree" electrocatalysts for ORR, it should be pointed out that the specific catalytic sites as well as the catalytic mechanisms are still unclear. Moreover, because the starting material GO contains metallic impurities brought by its preparation method and it has been demonstrated that even a slight trace of Mn metallic impurities in graphene materials is sufficient to alter or dominate their electrocatalytic properties towards ORR, Wang and coworkers argued that the "metal-free" electrocatalysis of the ORR on heteroatomdoped graphene is caused by trace levels ( $\mathrm{ppm}$ ) of metallic impurities (manganese oxide in particular) present within the graphene materials [43]. Therefore, more future works are encouraged to study and prove that the doping atoms other than the residual metallic impurities in these 3D GF played a dominant role in the electrocatalysis of the ORR on electrodes.

3.2. Metal-Free 3D Graphene-Based Nanocomposites. As mentioned above, $\mathrm{N}$-doped carbon materials have been reported to be efficient metal-free electrocatalysts for ORR; however, the low level of nitrogen concentration limited by their preparation methods may affect further improvements in

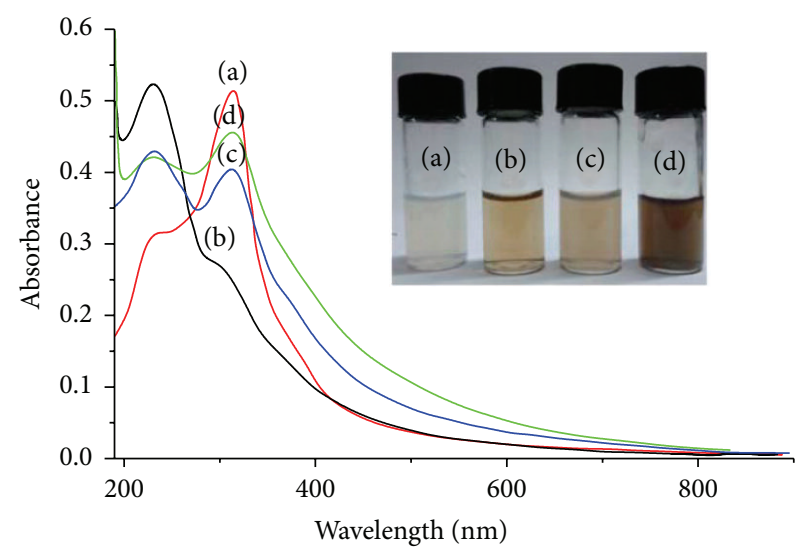

FIGURE 3: UV-vis absorption spectra and corresponding photographs (inset) of aqueous dispersions of $g-\mathrm{C}_{3} \mathrm{~N}_{4}$ nanosheets (a), $\mathrm{GO}(\mathrm{b}), \mathrm{g}-\mathrm{C}_{3} \mathrm{~N}_{4} / \mathrm{GO}(\mathrm{c})$, and $\mathrm{g}-\mathrm{C}_{3} \mathrm{~N}_{4} / \mathrm{rGO}$ (reprinted from [44]; copyright 2014 American Chemical Society).

their catalytic performances. Graphitic carbon nitride (g$\mathrm{C}_{3} \mathrm{~N}_{4}$ ), which possesses high nitrogen content including both pyridinic and graphitic nitrogen moieties which could be potential active sites for ORR, is thus considered to be a potential catalyst or an effective component in composite catalyst for ORR.

In 2014, Tian and coworkers constructed a novel 3D porous supramolecular architecture of ultrathin $\mathrm{g}-\mathrm{C}_{3} \mathrm{~N}_{4}$ nanosheets and $\mathrm{rGO}\left(\mathrm{g}-\mathrm{C}_{3} \mathrm{~N}_{4} / \mathrm{rGO}\right)$ by solution self-assembly of $\mathrm{g}^{-} \mathrm{C}_{3} \mathrm{~N}_{4}$ and $\mathrm{GO}$ followed by photoreduction catalyzed by g- $\mathrm{C}_{3} \mathrm{~N}_{4}$ (Figure 2) [44]. The $\pi$-stacking interactions between the $\mathrm{sp}^{2}$ lattice of $\mathrm{g}-\mathrm{C}_{3} \mathrm{~N}_{4}$ and the $\mathrm{sp}^{2}$ graphene lattice as well as the hydrogen-bonding interactions between the $\mathrm{N}$ containing groups in $\mathrm{g}-\mathrm{C}_{3} \mathrm{~N}_{4}$ and $\mathrm{O}$ functional groups of $\mathrm{GO}$ were believed to be responsible for the self-assembly formation of the $3 \mathrm{D}$ porous frameworks in solution - this presumption could be further confirmed by the shift of the UV-vis absorption band of $\mathrm{g}^{-} \mathrm{C}_{3} \mathrm{~N}_{4}$ in the presence of $\mathrm{GO}$ (Figure 3). The as-prepared g- $\mathrm{C}_{3} \mathrm{~N}_{4} / \mathrm{rGO}$ hybrid possessed high surface area, multilevel porous structure, good electrical conductivity, efficient electron transport network, and fast charge transfer kinetics at $g-\mathrm{C}_{3} \mathrm{~N}_{4} / \mathrm{rGO}$ interfaces, which facilitate the diffusion of $\mathrm{O}_{2}$, electrolyte, and electrons in the porous frameworks during ORR. As a novel ORR catalyst, g- $\mathrm{C}_{3} \mathrm{~N}_{4} / \mathrm{rGO}$ 


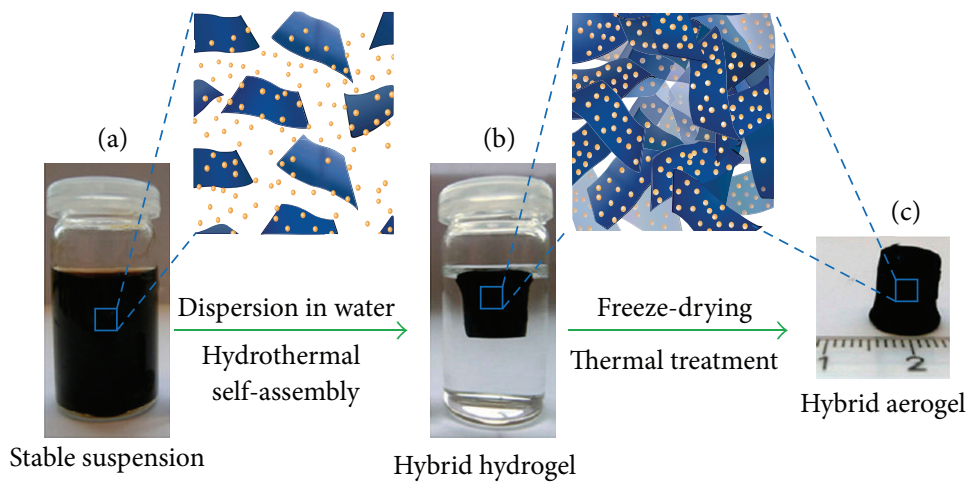

Figure 4: Fabrication process for the 3D $\mathrm{Fe}_{3} \mathrm{O}_{4} / \mathrm{N}$-GAs catalyst. (a) Stable suspension of GO, iron ions, and PPy dispersed in a vial. (b) Feand PPy-supporting graphene hybrid hydrogel prepared by hydrothermal self-assembly and floating on water in a vial and its ideal assembled model. (c) Monolithic $\mathrm{Fe}_{3} \mathrm{O}_{4} / \mathrm{N}-\mathrm{GAs}$ hybrid aerogel obtained after freeze-drying and thermal treatment (reprinted from [46]; copyright 2012 American Chemical Society).

exhibited superior ORR catalytic activity over single component, physical mixed or in situ immobilized composite or previously reported $\mathrm{g}-\mathrm{C}_{3} \mathrm{~N}_{4} / \mathrm{C}$ composites in alkaline mediums. In comparison with $\mathrm{Pt} / \mathrm{C}$ catalyst, g- $\mathrm{C}_{3} \mathrm{~N}_{4} / \mathrm{rGO}$ showed comparable ORR catalytic activity, higher stability, and better tolerance toward methanol poisoning, with a fourelectron dominated ORR process. This work exemplifies a new way to prepare metal-free, $3 \mathrm{D}$ graphene-based composite as ORR catalyst which combines the advantages of $3 \mathrm{D}$ graphene (high surface area, good electrical conductivity, etc.) with those of metal-free catalysts (sustainability, low cost, etc.).

Besides the aforementioned metal-free 3D graphene/ inorganic composite as ORR catalyst, Zhuang and coworkers reported a graphene/biofilm composite with a $3 \mathrm{D}$ structure as biocathode in microbial fuel cell (MFC) [45]. This composite was fabricated by a novel one-pot method that the microbially reduced graphene was implanted into cathode biofilm, leading to the in situ construction of graphene/biofilm composite. As an effective biocathode catalyst for ORR, this graphene/biofilm composite could enhance the electrocatalytic activity and facilitate the electron transfer between bacteria and electrode, thus showing higher maximum power density of MFC, faster electron transfer kinetics, and less internal resistance as compared with those of carbon clothbiocathode.

3.3. Nonprecious Metal-Containing 3D Graphene-Based Nanocomposites. As alternative ORR catalysts for Pt-based materials, nonprecious metals ( $\mathrm{Fe}, \mathrm{Co}$, etc.) or metal oxides $\left(\mathrm{Fe}_{3} \mathrm{O}_{4}\right.$, $\mathrm{Co}_{3} \mathrm{O}_{4}$, etc.) have been actively studied; however, these catalysts tend to degrade during operation of the fuel cell due to dissolution, sintering, and agglomeration. Supporting these nonprecious metal/metal oxide nanoparticles (NPs) on $3 \mathrm{D}$ graphene networks would be an attractive way to solve these problems, as a geometric confinement of these NPs within graphene layers would enhance their interface contact and suppress the dissolution and agglomeration of NPs, thereby promoting the electrochemical activity and stability of the hybrids.
In 2012, $\mathrm{Wu}$ and coworkers reported 3D $\mathrm{N}$-doped graphene aerogel- (N-GA-) supported $\mathrm{Fe}_{3} \mathrm{O}_{4}$ nanoparticles $\left(\mathrm{Fe}_{3} \mathrm{O}_{4} / \mathrm{N}-\mathrm{GAs}\right)$ as efficient cathode catalysts for ORR [46]. These 3D monolithic $\mathrm{Fe}_{3} \mathrm{O}_{4} / \mathrm{N}-\mathrm{GAs}$ hybrids were fabricated via a combined hydrothermal self-assembly, freeze-drying, and thermal treatment process, using GO, iron acetate, and polypyrrole as starting materials (Figure 4). Because of the 3D macroporous structure, high surface area, and uniform deposition of $\mathrm{Fe}_{3} \mathrm{O}_{4} \mathrm{NPs}$, the resulting $\mathrm{Fe}_{3} \mathrm{O}_{4} / \mathrm{N}-\mathrm{GAs}$ exhibited a more positive onset potential, higher cathodic density, lower $\mathrm{H}_{2} \mathrm{O}_{2}$ yield, and higher electron transfer number $\left(4 \mathrm{e}^{-}\right)$ for the ORR in alkaline media than $\mathrm{Fe}_{3} \mathrm{O}_{4}$ NPs supported on $\mathrm{N}$-doped carbon black or $\mathrm{N}$-doped 2D graphene sheets. Furthermore, $\mathrm{Fe}_{3} \mathrm{O}_{4} / \mathrm{N}$-GAs showed better durability than the commercial Pt/C catalyst. This 3D N-GA is also demonstrated to be a universal carbon support that is superior to other carbon supports for loading of other nonprecious metal electrocatalysts (e.g., Fe NPs).

Iron porphyrin plays a vital role in oxygen transport and reduction reactions in biological system. Thus, it would be attractive to consider if supporting iron porphyrin on graphene could function as an alternative to Pt-based electrode in fuel cells for ORR. In 2012, Jahan and coworkers synthesized an electrocatalytically active 3D graphene-iron porphyrin metal organic framework (MOF) composite for ORR by reacting the pyridine-functionalized graphene with iron porphyrin [47]. According to their study, the addition of functionalized rGO sheets influenced the crystallization process of MOF, increased its porosity and electroactive surface area, and enhanced the electrochemical charge transfer rate of iron porphyrin. The synergistic effect of the rGO, pyridinium linker, and the iron porphyrin for the improvements in electrocatalytic activities was demonstrated by a series of control experiments. With a four-electron ORR pathway, this graphene-metalloporphyrin hybrid also possessed significantly reduced methanol crossover effects and superior durability compared to $\mathrm{Pt}$ catalysts, showing its potential for use as a promising Pt-free cathode in alkaline direct methanol fuel cell. 


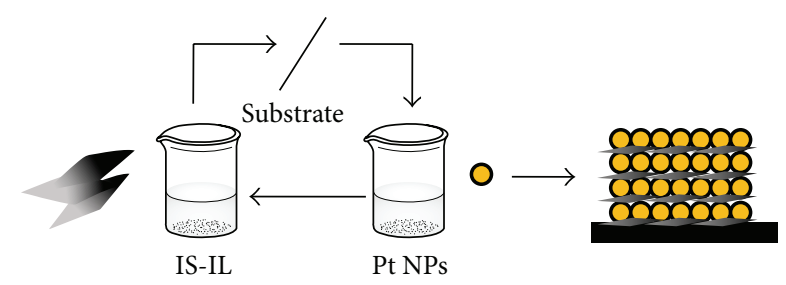

FIGURE 5: Schematic representation of the assembling process of the IS-IL modified graphene/Pt NPs multilayer films (reprinted from [49]; copyright 2010 American Chemical Society).

Recently, Mao and coworkers reported a 3D N-doped crumpled graphene- (N-CG-) cobalt oxide nanohybrids (NCG-CoO) with a stable structure for use as bifunctional catalysts for both ORR and oxygen evolution reaction (OER) in alkaline solutions [48]. The N-CG-CoO nanocrystal hybrid, showing a hollow structure with uniform nanocrystal decoration on both the external and internal surfaces of the CG balls, was produced by an aerosolization/high-temperatureinduced GO crumpling and nanocrystal growth method. With a four-electron ORR process, the N-CG-CoO hybrid exhibited excellent ORR catalytic activity that is comparable with that of commercial $\mathrm{Pt} / \mathrm{C}$ catalyst (and is much higher than that of nondoped CG-CoO, rGO, and $\mathrm{CoO}$ ) and good durability superior to that of $\mathrm{Pt} / \mathrm{C}$ in an alkaline electrolyte and thus is believed to be a promising candidate for fuel cell and water splitting applications.

\subsection{Noble Metal-Containing 3D Graphene-Based Nanocom-} posites. Although great contribution has been made to devise various nonnoble metal electrocatalysts, the demands of the high catalytic activity for the four-electron ORR and the acidic environment of the fuel cell electrode still make noble metal-based nanostructures the most promising for practical application. In this context, it is a realistic strategy to further maximize the activity of noble metal-based nanostructures and minimize the use of precious noble metal by loading these highly active nanomaterials on the surface of supporting materials with low cost, high surface area, and good electrical conductivity, which not only maximizes the availability of surface area for electron transfer and decreases the aggregation of these electrocatalysts, but also provides better mass transport of reactants to the electrocatalysts. As expected, 3D graphene network, among the catalyst supports for ORR, is believed to be the most promising candidate owing to its unique properties such as high electrical and thermal conductivities, great mechanical strength, inherent flexibility, and huge specific surface area.

In 2010, Zhu and coworkers constructed a hybrid 3D nanocomposite film by alternatively assembling the graphene nanosheets modified by imidazolium salt-based ionic liquid (IS-IL) and Pt NPs through electrostatic interaction (Figure 5) [49]. The introduction of IS-IL on the surface of graphene nanosheets not only increases the dissolubility of graphene nanosheets, but also provides the catalyst support with higher conductivity and abundant positive charge desired in this layer-by-layer (LBL) self-assembly of nanocomposites. This multilayer film exhibited prominent electrocatalytic activity and stability toward ORR. Furthermore, the content of Pt NPs-and thus the electrocatalytic activity-of the films could be tailored by tuning the number of bilayers in the LBL process.

In 2013, Sattayasamitsathit and coworkers reported for the first time on the use of lithographically defined 3D graphene microstructure as support for catalytic metal NPs (Figure 6) [50]. Various noble metal NPs ( $\mathrm{Au}, \mathrm{Pd}$, and $\mathrm{Pt}$ ) were deposited on these multilayered highly ordered 3D graphene structures by either electrochemical or electroless method. The size, morphology, and distribution of the NPs over the $3 \mathrm{D}$ graphene structure could be tailored by changing the preparation conditions (e.g., deposition potential or time for electrochemical method; metal concentration, $\mathrm{pH}$, or temperature for chemical reduction method). The catalytic activity toward ORR of Pt NPs on 3D graphene was superior to that of Pt NPs on a 3D carbon substrate and on a glassy carbon electrode, bare 3D graphene, and Pt black. This enhancement of catalytic activity could be ascribed to the macroporosity of 3D graphene which allows deposition of a high surface area of catalytic NPs and the nanosize dimensions of the connecting arms and nodes in 3D graphene structure which provides a highly favorable mass transport environment for delivery of fuels.

\section{Conclusions and Perspective}

In fuel cells, an effective electrocatalyst for ORR at the cathode plays a crucial role in improving their energy conversion efficiency and enhancing the high energy and power density. In recent years, the newly developed 3D graphene-based materials have been demonstrated to be promising as efficient electrocatalysts for ORR. In this paper, we have reviewed the primary preparation method of these novel materials and their performances as catalysts for ORR. From the viewpoint of whether containing precious metals or not, Sections 3.13.3 have introduced the development of noble metal-free 3D graphene-based electrocatalysts, which is among the current efforts to develop the alternative to Pt-based catalysts for ORR. The success in this direction would significantly weaken or fully eliminate the dependency on noble metals, thereby greatly reducing the cost of fuel cells. On the other hand, Section 3.4 has introduced the development of low-noble metal 3D graphene-based electrocatalysts, which belongs to another class of efforts to maximize the utilization of noble metal in fuel cells application. As mentioned earlier, 3D graphene could function as either single catalyst or catalyst support for ORR. As the single catalyst, heteroatomdoped 3D graphene-based materials could render stable and durable catalytic performance compared with other composite electrocatalysts for ORR. As the catalysts support, compared to another carbonaceous support, 3D graphene combines the advantages of traditional 2D graphene (such as high conductivity and large specific surface area) with its nonagglomerated morphology and porous structure, which could facilitate the loading and dispersion of catalysts. Moreover, the interconnected graphene networks could promote fast electron transport between the reaction sites and 


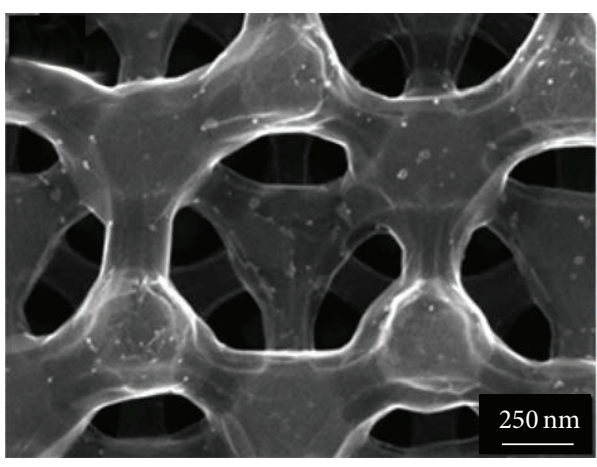

(a)

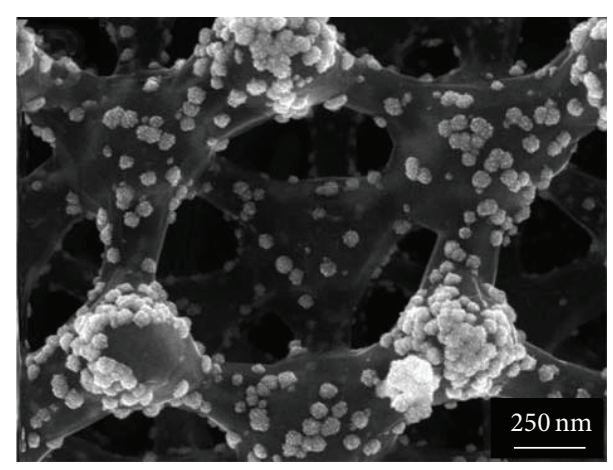

(b)

FIGURE 6: SEM images of (a) bare 3D graphene substrate before modification with metal nanoparticles and (b) 3D graphene after modification with Pt NPs using electrodeposition at $0.4 \mathrm{~V}$ for $750 \mathrm{~s}$ (reproduced from [50] with permission of the Royal Society of Chemistry).

the electrode and efficiently maximize the electroactive surface area of catalysts, all of which would greatly improve the catalytic activity and durability of these 3D graphene-based electrocatalysts for ORR [51-54].

In spite of significant progress made in this area within only several years, the research of $3 \mathrm{D}$ graphene-based materials still remains at its initial stage and at least two aspects of challenges for practical application of these materials in fuel cells should be addressed. First, the systematic exploration and deeper understanding of the different catalytic mechanisms of various 3D graphene-based materials for ORR are required for optimum material design and device performance optimization. For example, for $\mathrm{N}$-doped $3 \mathrm{D}$ graphene as inherent ORR catalyst, though the critical role of $\mathrm{N}$ dopant for the catalytic enhancement is proved, the precise relationship between catalytic activity and $\mathrm{N}$ species is still controversial, while, for 3D-graphene-based nanocomposites, more experimental and theoretical studies are needed to reveal the interactions between the $3 \mathrm{D}$ graphene and loaded active nanomaterials. Second, the development of facile, green, cost-effective, and controllable preparation method is still a significant issue, because the current assembly process usually needs to be carried out in rigorous conditions (such as high temperature or pressure) or involves tedious procedures, which increases the degree of preparation difficulty. Thus, much further research is needed to realize the aim of final industrial implementation, large scale, low cost, and simple production of 3D graphene-based materials with high catalytic activity and practical durability for ORR. This review is anticipated to encourage more future works to achieve this aim and to promote sustainability.

\section{Conflict of Interests}

The authors declare that there is no conflict of interests regarding the publication of this paper.

\section{Acknowledgments}

This work was supported by the Natural Science Foundation of Shanxi (no. 2015021081), the Project for Construction of
Science and Technology Innovation Team of Shanxi (no. 2015013001-04), and the Provincial Science and Technology Major Project of Shanxi (no. 0111101059).

\section{References}

[1] V. Dhand, K. Y. Rhee, H. Ju Kim, and D. Ho Jung, "A comprehensive review of graphene nanocomposites: research status and trends," Journal of Nanomaterials, vol. 2013, Article ID 763953, 14 pages, 2013.

[2] A. K. Geim and K. S. Novoselov, "The rise of graphene," Nature Materials, vol. 6, no. 3, pp. 183-191, 2007.

[3] K. S. Novoselov, V. I. Fal'Ko, L. Colombo, P. R. Gellert, M. G. Schwab, and K. Kim, "A roadmap for graphene," Nature, vol. 490, no. 7419, pp. 192-200, 2012.

[4] K. S. Novoselov, A. K. Geim, S. V. Morozov et al., "Electric field effect in atomically thin carbon films," Science, vol. 306, no. 5696, pp. 666-669, 2004.

[5] X. Huang, X. Qi, F. Boey, and H. Zhang, "Graphene-based composites," Chemical Society Reviews, vol. 41, no. 2, pp. 666-686, 2012.

[6] X. Zhang, K. S. Ziemer, K. Zhang et al., "Large-area preparation of high-quality and uniform three-dimensional graphene networks through thermal degradation of graphene oxidenitrocellulose composites," ACS Applied Materials and Interfaces, vol. 7, no. 2, pp. 1057-1064, 2015.

[7] Y. Zhu, S. Murali, W. Cai et al., "Graphene and graphene oxide: synthesis, properties, and applications," Advanced Materials, vol. 22 , no. 35, pp. 3906-3924, 2010.

[8] X. Zhang, X. Ji, R. Su, B. L. Weeks, Z. Zhang, and S. Deng, "Aerobic oxidation of $9 \mathrm{~h}$-fluorenes to 9-fluorenones using mono-/multilayer graphene-supported alkaline catalyst," ChemPlusChem, vol. 78, no. 7, pp. 703-711, 2013.

[9] A. Aziz, H. N. Lim, S. H. Girei et al., "Silver/graphene nanocomposite-modified optical fiber sensor platform for ethanol detection in water medium," Sensors and Actuators B: Chemical, vol. 206, pp. 119-125, 2015.

[10] S. P. Lim, A. Pandikumar, Y. S. Lim, N. M. Huang, and H. N. Lim, "In-situ electrochemically deposited polypyrrole nanoparticles incorporated reduced graphene oxide as an efficient counter electrode for platinum-free dye-sensitized solar cells," Scientific Reports, vol. 4, article 5305, 2014. 
[11] G. T. S. How, A. Pandikumar, H. N. Ming, and L. H. Ngee, "Highly exposed 001 facets of titanium dioxide modified with reduced graphene oxide for dopamine sensing," Scientific Reports, vol. 4, article 5044, 2014.

[12] K. Zangeneh Kamali, A. Pandikumar, G. Sivaraman et al., "Silver@graphene oxide nanocomposite-based optical sensor platform for biomolecules," RSC Advances, vol. 5, no. 23, pp. 17809-17816, 2015.

[13] M. M. Shahid, A. Pandikumar, A. M. Golsheikh, N. M. Huang, and H. N. Lim, "Enhanced electrocatalytic performance of cobalt oxide nanocubes incorporating reduced graphene oxide as a modified platinum electrode for methanol oxidation," $R S C$ Advances, vol. 4, no. 107, pp. 62793-62801, 2014.

[14] A. Pandikumar, G. T. S. How, T. P. See et al., "Graphene and its nanocomposite material based electrochemical sensor platform for dopamine," RSC Advances, vol. 4, no. 108, pp. 63296-63323, 2014.

[15] J. K. Gan, Y. S. Lim, A. Pandikumar, N. M. Huang, and H. N. Lim, "Graphene/polypyrrole-coated carbon nanofiber coreshell architecture electrode for electrochemical capacitors," RSC Advances, vol. 5, no. 17, pp. 12692-12699, 2015.

[16] F. A. Jumeri, H. N. Lim, Z. Zainal, N. M. Huang, A. Pandikumar, and S. P. Lim, "Dual functional reduced graphene oxide as photoanode and counter electrode in dye-sensitized solar cells and its exceptional efficiency enhancement," Journal of Power Sources, vol. 293, pp. 712-720, 2015.

[17] S. P. Lim, A. Pandikumar, N. M. Huang, and H. N. Lim, "Reduced graphene oxide-titania nanocomposite-modified photoanode for efficient dye-sensitized solar cells," International Journal of Energy Research, vol. 39, no. 6, pp. 812-824, 2015.

[18] T. Peik-See, A. Pandikumar, L. H. Ngee, H. N. Ming, and C. C. Hua, "Magnetically separable reduced graphene oxide/iron oxide nanocomposite materials for environmental remediation," Catalysis Science \& Technology, vol. 4, no. 12, pp. 43964405, 2014.

[19] B. Xia, Y. Yan, X. Wang, and X. W. Lou, "Recent progress on graphene-based hybrid electrocatalysts," Materials Horizons, vol. 1, no. 4, pp. 379-399, 2014.

[20] C. Huang, C. Li, and G. Shi, “Graphene based catalysts," Energy \& Environmental Science, vol. 5, no. 10, pp. 8848-8868, 2012.

[21] B. F. MacHado and P. Serp, "Graphene-based materials for catalysis," Catalysis Science and Technology, vol. 2, no. 1, pp. 5475, 2012.

[22] F. Liu, C. W. Lee, and J. S. Im, "Graphene-based carbon materials for electrochemical energy storage," Journal of Nanomaterials, vol. 2013, Article ID 642915, 11 pages, 2013.

[23] L. Chen, M. Zhang, and W. Wei, "Graphene-based composites as cathode materials for lithium ion batteries," Journal of Nanomaterials, vol. 2013, Article ID 940389, 8 pages, 2013.

[24] X. Cao, Z. Yin, and H. Zhang, “Three-dimensional graphene materials: preparation, structures and application in supercapacitors," Energy and Environmental Science, vol. 7, no. 6, pp. 1850-1865, 2014.

[25] B. Luo and L. Zhi, "Design and construction of three dimensional graphene-based composites for lithium ion battery applications," Energy \& Environmental Science, vol. 8, no. 2, pp. 456477, 2015.

[26] S. Mao, G. Lu, and J. Chen, "Three-dimensional graphene-based composites for energy applications," Nanoscale, vol. 7, no. 16, pp. 6924-6943, 2015.
[27] S. Nardecchia, D. Carriazo, M. L. Ferrer, M. C. Gutiérrez, and F. Del Monte, "Three dimensional macroporous architectures and aerogels built of carbon nanotubes and/or graphene: synthesis and applications," Chemical Society Reviews, vol. 42, no. 2, pp. 794-830, 2013.

[28] Y. Shen, Q. Fang, and B. Chen, "Environmental applications of three-dimensional graphene-based macrostructures: adsorption, transformation, and detection," Environmental Science Technology, vol. 49, no. 1, pp. 67-84, 2015.

[29] J. Zai and X. Qian, “Three dimensional metal oxides-graphene composites and their applications in lithium ion batteries," RSC Advances, vol. 5, no. 12, pp. 8814-8834, 2015.

[30] Y. J. Wang, N. Zhao, B. Fang, H. Li, X. T. Bi, and H. Wang, "Carbon-supported pt-based alloy electrocatalysts for the oxygen reduction reaction in polymer electrolyte membrane fuel cells: particle size, shape, and composition manipulation and their impact to activity," Chemical Reviews, vol. 115, no. 9, pp. 3433-3467, 2015.

[31] C. Wang, N. M. Markovic, and V. R. Stamenkovic, "Advanced platinum alloy electrocatalysts for the oxygen reduction reaction," ACS Catalysis, vol. 2, no. 5, pp. 891-898, 2012.

[32] K. Chen, L. Chen, Y. Chen, H. Bai, and L. Li, “Three-dimensional porous graphene-based composite materials: electrochemical synthesis and application," Journal of Materials Chemistry, vol. 22, no. 39, pp. 20968-20976, 2012.

[33] Z. Yang, H. Nie, X. Chen, X. Chen, and S. Huang, "Recent progress in doped carbon nanomaterials as effective cathode catalysts for fuel cell oxygen reduction reaction," Journal of Power Sources, vol. 236, pp. 238-249, 2013.

[34] C. Zhu and S. Dong, "Recent progress in graphene-based nanomaterials as advanced electrocatalysts towards oxygen reduction reaction," Nanoscale, vol. 5, no. 5, pp. 1753-1767, 2013.

[35] Q. Fang, Y. Shen, and B. Chen, "Synthesis, decoration and properties of three-dimensional graphene-based macrostructures: a review," Chemical Engineering Journal, vol. 264, pp. 753-771, 2015.

[36] C. Li and G. Shi, “Three-dimensional graphene architectures," Nanoscale, vol. 4, no. 18, pp. 5549-5563, 2012.

[37] Z. Yan, W. Yao, L. Hu, D. Liu, C. Wang, and C. Lee, "Progress in the preparation and application of three-dimensional graphenebased porous nanocomposites," Nanoscale, vol. 7, no. 13, pp. 5563-5577, 2015.

[38] M. Zeng, W.-L. Wang, and X.-D. Bai, "Preparing three-dimensional graphene architectures: review of recent developments," Chinese Physics B, vol. 22, no. 9, Article ID 098105, 2013.

[39] X.-K. Kong, C.-L. Chen, and Q.-W. Chen, "Doped graphene for metal-free catalysis," Chemical Society Reviews, vol. 43, no. 8, pp. 2841-2857, 2014.

[40] H. Wang, T. Maiyalagan, and X. Wang, "Review on recent progress in nitrogen-doped graphene: synthesis, characterization, and its potential applications," ACS Catalysis, vol. 2, no. 5, pp. 781-794, 2012.

[41] Y. Zhao, C. Hu, Y. Hu, H. Cheng, G. Shi, and L. Qu, "A versatile, ultralight, nitrogen-doped graphene framework," Angewandte Chemie International Edition, vol. 51, no. 45, pp. 11371-11375, 2012.

[42] Y. Su, Y. Zhang, X. Zhuang et al., "Low-temperature synthesis of nitrogen/sulfur co-doped three-dimensional graphene frameworks as efficient metal-free electrocatalyst for oxygen reduction reaction," Carbon, vol. 62, pp. 296-301, 2013. 
[43] L. Wang, A. Ambrosi, and M. Pumera, “Metal-free' catalytic oxygen reduction reaction on heteroatom-doped graphene is caused by trace metal impurities," Angewandte ChemieInternational Edition, vol. 52, no. 51, pp. 13818-13821, 2013.

[44] J. Tian, R. Ning, Q. Liu, A. M. Asiri, A. O. Al-Youbi, and X. Sun, "Three-dimensional porous supramolecular architecture from ultrathin $g-\mathrm{C}_{3} \mathrm{~N}_{4}$ nanosheets and reduced graphene oxide: solution self-assembly construction and application as a highly efficient metal-free electrocatalyst for oxygen reduction reaction," ACS Applied Materials \& Interfaces, vol. 6, no. 2, pp. 10111017, 2014.

[45] L. Zhuang, Y. Yuan, G. Yang, and S. Zhou, "In situ formation of graphene/biofilm composites for enhanced oxygen reduction in biocathode microbial fuel cells," Electrochemistry Communications, vol. 21, no. 1, pp. 69-72, 2012.

[46] Z.-S. Wu, S. Yang, Y. Sun, K. Parvez, X. Feng, and K. Müllen, “3D nitrogen-doped graphene aerogel-supported $\mathrm{Fe}_{3} \mathrm{O}_{4}$ nanoparticles as efficient electrocatalysts for the oxygen reduction reaction," Journal of the American Chemical Society, vol. 134, no. 22, pp. 9082-9085, 2012.

[47] M. Jahan, Q. Bao, and K. P. Loh, "Electrocatalytically active graphene-porphyrin MOF composite for oxygen reduction reaction," Journal of the American Chemical Society, vol. 134, no. 15, pp. 6707-6713, 2012.

[48] S. Mao, Z. Wen, T. Huang, Y. Hou, and J. Chen, "Highperformance bi-functional electrocatalysts of 3D crumpled graphene-cobalt oxide nanohybrids for oxygen reduction and evolution reactions," Energy \& Environmental Science, vol. 7, no. 2, pp. 609-616, 2014.

[49] C. Zhu, S. Guo, Y. Zhai, and S. Dong, "Layer-by-layer selfassembly for constructing a graphene/platinum nanoparticle three-dimensional hybrid nanostructure using ionic liquid as a linker," Langmuir, vol. 26, no. 10, pp. 7614-7618, 2010.

[50] S. Sattayasamitsathit, Y. Gu, K. Kaufmann et al., "Highly ordered multilayered 3D graphene decorated with metal nanoparticles," Journal of Materials Chemistry A, vol. 1, no. 5, pp. 1639-1645, 2013.

[51] D. Geng, N. Ding, T. S. Andy Hor, Z. Liu, X. Sun, and Y. Zong, "Potential of metal-free 'graphene alloy' as electrocatalysts for oxygen reduction reaction," Journal of Materials Chemistry A, vol. 3, no. 5, pp. 1795-1810, 2015.

[52] D.-W. Wang and D. Su, "Heterogeneous nanocarbon materials for oxygen reduction reaction," Energy \& Environmental Science, vol. 7, no. 2, pp. 576-591, 2014.

[53] Y. Nie, L. Li, and Z. Wei, "Recent advancements in Pt and Ptfree catalysts for oxygen reduction reaction," Chemical Society Reviews, vol. 44, no. 8, pp. 2168-2201, 2015.

[54] L. Dai, Y. Xue, L. Qu, H.-J. Choi, and J.-B. Baek, "Metal-free catalysts for oxygen reduction reaction," Chemical Reviews, vol. 115, no. 11, pp. 4823-4892, 2015. 

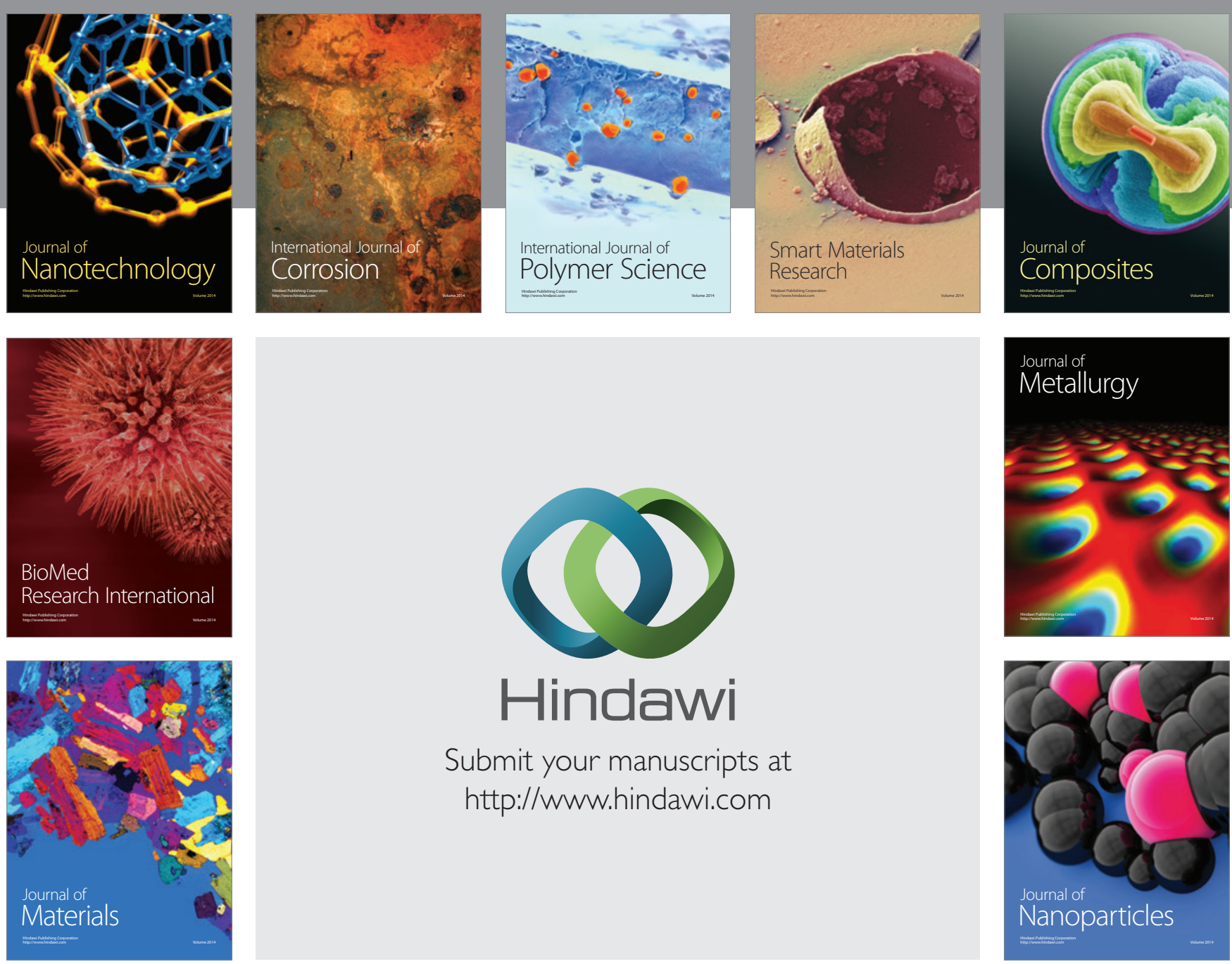

Submit your manuscripts at http://www.hindawi.com
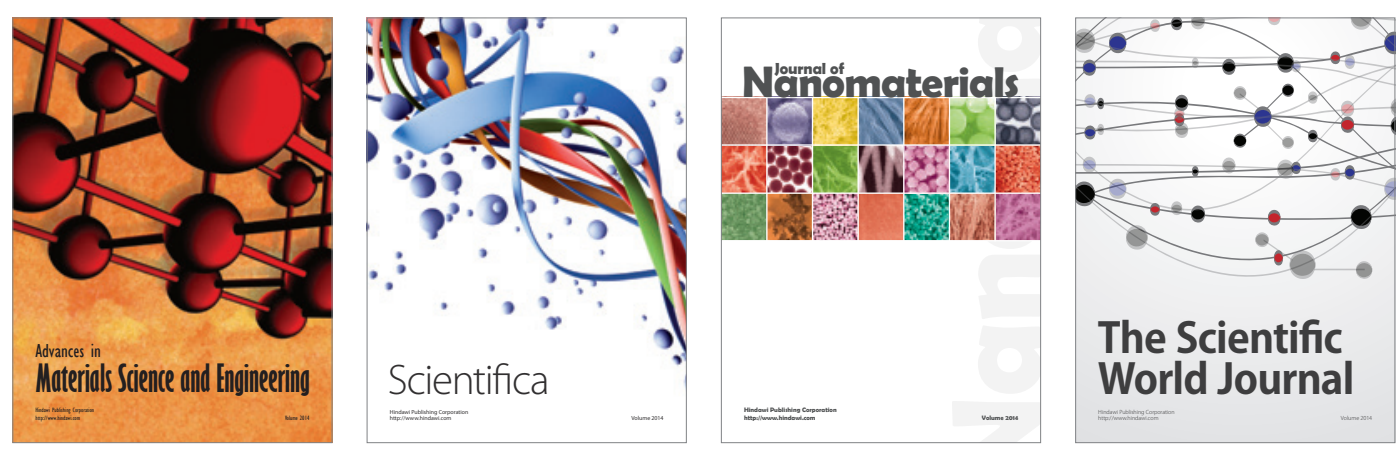

\section{The Scientific World Journal}
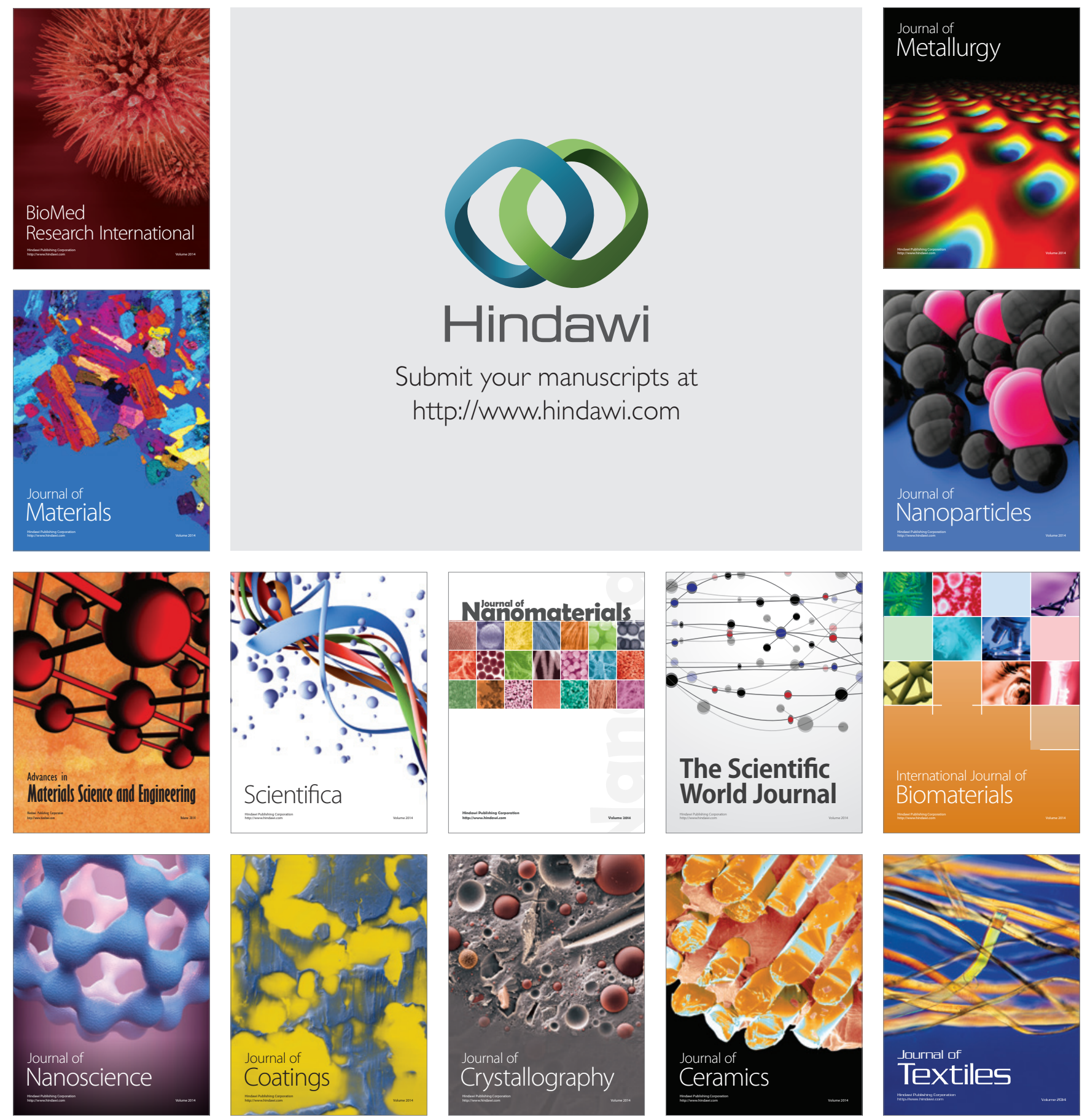